\title{
REFLEXIÓN ACERCA DEL ROL DEL DOCENTE UNIVERSITARIO Y EL FORTALECIMIENTO DEL PENSAMIENTO COMPLEJO EN ESTUDIANTES DE MAESTRÍA
}

Rossi Salinas ${ }^{1 *}$, Jaimen Fung ${ }^{2}$, Samaria Muñoz ${ }^{3}$

${ }^{1}$ ISAE Universidad. Departamento de Investigación y Postgrado. Panamá, República de Panamá.

${ }^{2}$ Team Fung Consultant Business, Investigador Pedagogo. Ecuador

${ }^{3}$ Universidad de Los Andes, Instituto de Investigaciones Económicas y Sociales, Mérida Venezuela.

*Autor para correspondencia: rossi.salinas@ujgh.edu.ve

Recibido: 2020/07/06

Aprobado: 2020/11/25

DOI: https://doi.org/10.26621/XVI23.2020.12.A03.PUCESI.2550.6684

\section{RESUMEN}

El presente artículo, tiene como objetivo general establecer la relación entre la reflexión del docente universitario y el fortalecimiento del pensamiento complejo en estudiantes de la maestría en docencia superior. Por lo que emerge la idea, si la reflexión del docente se sustenta en el ser, hacer y emprender de saberes, permitiendo así que brinde herramientas metacognitivas a los maestrantes para desarrollar la complejidad del pensamiento. Por lo cual, esta investigación se despliega sobre la episteme de diversos autores que permitieron sustentar teóricamente este compendio. En este sentido, la investigación se construye bajo el paradigma positivista, con un enfoque cuantitativo y de tipo correlacional, ya que tienen el propósito de medir el grado de relación que existe entre dos o más variables, de igual forma se aplica un diseño no experimental transaccional transversal de campo. La población está conformada por 45 estudiantes de la maestría en Docencia Superior de Institución Superior De Administración Y Educación, ISAE Universidad. Para la recolección de datos, se aplicó el cuestionario como instrumento dirigido a la muestra estudiada. La metodología para el procesamiento de datos fue la estadística descriptiva, mediante la distribución de frecuencias considerando la absoluta y la relativa. La correlación se efectuó a través de la fórmula de Pearson, dando como resultado una correlación medianamente positiva entre las variables reflexión del docente universitario y pensamiento complejo. Se concluyó que los docentes universitarios poseen y manejan conocimientos holísticos e integrales desde el ser hasta el emprender, sin embargo, se consideró pertinente sugerir actividades 0 acciones menos tradicionales para que el saber generado llegue de manera significativa a los estudiantes y estos activen la complejidad del pensamiento en la producción científica dentro del programa de maestría.

Palabras clave: reflexión rol docente, pensamiento complejo, nivel superior 


\section{ABSTRACT}

The general objective of this article is to establish the relationship between the reflection of the university teacher and the strengthening of complex thinking in students of the master's degree in higher education. The idea emerges, if the teacher's reflection is based on being, doing and undertaking knowledge, thus allowing teachers to provide metacognitive tools to develop the complexity of thinking. Therefore, this research unfolds on the episteme of various authors who allowed to theoretically support this compendium. In this regard the research is built under the positivist paradigm, with a quantitative and correlational approach, since they have the purpose of measuring the relationship degree that It exists between two or more variables, in the same way a non-experimental design is applied cross-field transactional. The population was made up of 45 students of the master's degree in Higher Teaching of the Higher Institution of Administration and Education, ISAE University. For data collection, the questionnaire was applied as an instrument directed to the sample study. The methodology for data processing was descriptive statistics, through the distribution of frequencies considering the absolute and the relative. The correlation was carried out through Pearson's formula, resulting in a moderately positive correlation between the variables reflection of the university professor and complex thinking. It was concluded that university teachers possess and handle holistic and comprehensive knowledge from being to undertaking, however, it was considered pertinent to suggest less traditional activities or actions for this, the knowledge generated reaches the students in a meaningful way and these activate the complexity of the thinking in scientific production within the master's program.

Keywords: reflection teaching role, complex thinking, higher level

\section{INTRODUCCIÓN}

Los investigadores, en su rol activo dentro de la maestría en docencia superior, han mostrado gran interés por saber que reflexiones asumiría el docente universitario para fortalecer el pensamiento complejo en los estudiantes de dicho programa. En este orden de idas, se destaca la relevancia de la investigación dentro del contexto socioeducativo y científico, dado que este articulo brinda las herramientas necesarias para comprender la praxis desde el hacer y emprender del docente, con el fin de facilitar a los participantes insumos que activen el análisis, la criticidad, la reflexión y por ende la complejidad del pensamiento. Recalcando, que es un requisito indispensable para los cursantes de estudios de cuarto nivel.

Núñez, et al., (2017), desarrollaron el pensamiento crítico en estudiantes universitarios por medio del Aprendizaje Basado en Problema y tuvo como propósito encontrar el beneficio de este tipo de aprendizaje en el desarrollo de las competencias genéricas del pensamiento crítico. Utilizando el método mixto con un diseño embebido y de tipo transeccional en un grupo de 27 personas de primer cuatrimestre de la materia de Introducción a la Nutrición y a la Dietética de la licenciatura en Nutrición, en una universidad privada de Iguala, Guerrero. Los instrumentos manejados fueron el cuestionario de competencias genéricas individuales, sección de pensamiento crítico y las rejillas de observación para las habilidades del pensamiento crítico. Desarrollado por (Facione, 1990: 15), citado en (Olivares y Heredia 2012), donde sus resultados mostraron que la técnica indicada, presentando un efecto real en las destrezas coherentes al juicio de un escenario específico, con datos objetivos y subjetivos.

De igual forma Rivera, et al., (2017), con un estudio similar y con aportes sustanciales denominado, la investigación científica en las universidades ecuatorianas, se expone el rol de la educación superior en el desarrollo de la producción científica en las universidades ecuatorianas, antes y después de instituido el modelo educativo vigente desde el año 2008, el cual visualiza la institución como un agente generador de cambio social, cultural y ambiental, en una lógica de pensamiento complejo e integrador. Mediante una indagación exploratoria y descriptiva, se constató que el sistema académico en el Ecuador, durante décadas, tuvo un enfoque docente diferente al aplicado en la actualidad. Se presentan los logros alcanzados, fundamentalmente, en la producción científica de las universidades y en el nivel académico de su claustro, y se enfatiza en la necesidad de implementar estrategias que permitan eliminar las debilidades existentes, a fin de lograr el reconocimiento internacional.

Considerando los estudios de Núñez et al., (2017) y Rivera et al., (2017), ambos del mismo año consideran que el accionar del docente es un elemento fundamental para la complementariedad en la adquisición de las competencias específicas y profesionales de los maestrantes del programa en docencia superior De allí, se hace necesario la auto reflexión en cuanto a la formación académica que practican los docentes universitarios para contribuir en la gestación de capacidades y la transformación de un pensamiento disgregado a uno más complejo, permitiendo ver los acontecimientos como un "holo" de manera, que los saberes académicos que se socializan dentro del programa de docencia superior, puedan innovar desde el hacer y emprender, llevando este a una metamorfosis de nuevos contenidos para replicarlos de forma holística y desprendida con la sociedad científica y educativa en general.

Cabe mencionar que la educación universitaria deja ver; que el conocimiento del docente universitario no se halla sujeto a un estándar específico; al contrario, este debe ser interactivo y proactivo, dirigido hacia las nuevas tendencias incluyendo todos aquellos elementos pluriculturales que se encuentra en los diversos espacios. De allí, que se piensa al facilitador como un gestor de conocimientos dinámicos que permita articular la realidad teórica con la realidad contextual y por ende llegar a construir un pensamiento holístico e integrativo.

En este sentido, la productividad del conocimiento asimilado por el docente universitario garantiza el desarrollo de las dimensiones humanas 
tales como el ser, hacer, conocer, y emprender a través de la praxis hasta producir o construir la complejidad del saber dentro o fuera de los recintos académicos de la educación superior.

La educación superior para el fortalecimiento del pensamiento complejo: En correspondencia con lo planteado, la UNESCO, a través del Proyecto Regional Educativo para la América Latina y el Caribe (PRELAC, 2013), confirma la voluntad de querer lograr en el ser humano el bienestar individual y colectivo como sociedad; por ello, se pone al descubierto hoy más que nunca el carácter estratégico de la educación innovadora y estratégica como herramienta para sumar valor a la sociedad.

Sin embargo, se observa cierta discrepancia entre lo teórico y la praxis que asumen los docentes universitarios, ya que se observa que pocos se toman el tiempo para reflexionar sobre su accionar académico. En este sentido se considera pertinente citar a Bustamante, Ayllón y Escanés. (2018), expresando que los docentes deben romper los esquemas tradicionales de su práctica cotidiana que confecciona en su hacer académico. Por ello, los investigadores mencionan unos principios básicos los cuales deben ser adoptados por el profesor universitario, dado que estos le permitirán el éxito en el fortalecimiento del pensamiento complejo como son el dialógico, recursivo y el hologramáticos, pues la triangulación de estos tres métodos combinados con las estrategias apropiadas generando la complementariedad del saber.

Construcción del pensamiento complejo: Se estructuran algunos de los conocimientos que deberían poseer los docentes universitarios, como punto de partida o de reflexión para la formación del pensamiento complejo de los estudiantes dentro de las aulas de clase.

Escrito o verbal: Tomando el aporte de Carlino (2002), la escritura no es considerada sólo un medio de expresar o comunicar el pensamiento elaborado previamente. Por el contrario, la concepción dominante es la que reconoce la función epistémica de la composición escrita: escribir permite incidir sobre el propio conocimiento a través de dos caminos. Por lo cual, es relevante considerar que el docente universitario debe manejar un discurso holístico, complejo, estructurado, técnico; sin embargo, el mismo se debe manejar de forma entendible para el estudiantado, ya que a través de la expresión verbal o escrita el participante construirá su propio juicio y establecerá su proceso de comprensión de la información.

Analítico y crítico: Esta permite orientar al estudiante desde la concepción del análisis del aprendizaje, así como el proceso de vinculación y producción cognitiva entre el docente y el discente. Puede afirmarse, que se genera con esto la empatía por la producción del conocimiento hasta llegar a la complejidad.

Tipos de Pensamiento: Mitológico, reflexivo y complejo: Estos tipos de pensamiento están involucrados con la evolución hacia los cambios fundamentales de los estilos de vida y los comportamientos tanto sociales como académicos del individuo, por lo cual la educación en su sentido más amplio- juega un papel preponderante. La educación es "la fuerza del futuro', porque, constituye uno de los instrumentos más poderosos para realizar el cambio. De esta manera, Morin (2010, p 81135) expresa, que el componente educativo es uno de los desafíos más difíciles de modificar y este implica el pensamiento holístico e integral, de manera que se enfrente la complejidad creciente, la rapidez de los cambios y lo impredecible que caracteriza al mundo.

\section{MATERIALES Y MÉTODOS}

Esta investigación se sustenta bajo el paradigma positivista con un enfoque cuantitativo, en línea con Fernández y Batista (2018), ya que este hace énfasis en la objetividad, orientada hacia los resultados. La investigación corresponde al tipo descriptivo de campo, correlacional, con diseño no experimental transaccional.

Las unidades de análisis fueron conformadas por cuarenta y cinco (45) estudiantes de la maestría en docencia superior de ISAE Universidad para dar respuesta al objetivo planteado; en la recolección de datos, se aplicó como instrumento un cuestionario dirigido a estudiantes, estructurados en 36 ítems, conformado por un modelo de escala tipo Likert con cinco (5) alternativas a escoger; dichas opciones fueron las siguientes: siempre, casi siempre, algunas veces, casi nunca y nunca.

En este caso, la aplicación de este instrumento se considera ajustada a los fines de la investigación, dado que permite discriminar las opiniones de los sujetos involucrados en el estudio, para responder a las interrogantes planteadas en la investigación. De allí que los valores resultantes de su aplicación serán de gran utilidad para que las respuestas obtenidas puedan cuantificarse e interpretarse a la luz de los objetivos de la investigación.

El instrumento aplicado fue validado por cuatro expertos en el área de Ciencias de la Educación. La confiabilidad se calculó a través de la siguiente fórmula:

$$
\mathrm{r}_{\mathrm{tt}}=\frac{\mathrm{K}}{\mathrm{K}-1}\left[1-\frac{\sum \mathrm{S}_{\mathrm{r}}^{2}}{\mathrm{~S}_{\mathrm{t}}{ }^{2}}\right]
$$

Donde:

$\mathrm{K}=$ Número de ítems.

$\mathrm{Sl}^{2}=$ Varianza de los puntajes de cada ítem.

$\mathrm{ST}^{2}=$ Varianza de los puntajes totales

$\mathrm{R}_{\mathrm{tt}}=$ Coeficiente de confiabilidad.

$1=$ Constante.

Los datos obtenidos como resultado de la prueba piloto fueron procesados a través del programa SPSS, en una matriz de doble entrada, para estimar el coeficiente de confiabilidad de los instrumentos, a fin de posteriormente aplicarlos a la población objeto de estudio. Una vez realizado el cálculo estadístico, se obtuvo la confiabilidad del instrumento de medición, arrojando un valor de 0,94, indicativo de que el cuestionario presenta una magnitud "muy alta" de acuerdo con el baremo propuesto. En este sentido, el instrumento guarda un coeficiente "muy alto". A continuación, se presenta una tabla que interpreta el valor del coeficiente Alfa de Cronbach de acuerdo con el rango y magnitud de análisis.

Tabla 1. Baremo de interpretación del coeficiente Alfa de Cronbach

\begin{tabular}{ll}
\hline \multicolumn{1}{c}{ Rango } & Categoría del Análisis \\
\hline $0,81-1,00$ & Muy Alta \\
\hline $0,61-0,80$ & Alta \\
\hline $0,41-0,60$ & Moderada \\
\hline $0,21-0,40$ & Bajo \\
\hline $0,01-0,20$ & Muy Baja \\
\hline
\end{tabular}

Fuente: Hernández y Mendoza. (2018) 
A tal efecto, se tabularon los valores recogidos, para confirmar que el instrumento es confiable en gran medida, lo cual permitió aplicarlo a la población en estudio. Para el procesamiento de los datos, se diseñó una tabla o matriz de doble entrada donde se asentaron los datos suministrados por los sujetos en atención a la sistematización de variables. Se utilizó la Estadística Descriptiva en lo que respecta a la distribución frecuencial y porcentual y el cálculo de las medias aritméticas, a través del programa para Estadística en Ciencias Sociales (SPSS) versión 19 en español, así como el programa EXCEL.

Posteriormente, para la discusión de los resultados, se elaboraron las tablas de distribución de medias aritméticas para los indicadores, dimensiones y variables; todo ello fue categorizado según un baremo previamente establecido que se construyó con base en los intervalos que ofrecen las alternativas de respuesta planteadas en cada instrumento. A continuación, se presenta el baremo diseñado con el rango y las categorías de análisis.

Tabla 2. Baremo de análisis

\begin{tabular}{ll}
\hline Rango & Categoría de análisis \\
\hline $4.5<5.00$ & Excelente \\
\hline $3.5<4.4$ & Bueno \\
\hline $2.5<3.4$ & Regular \\
\hline $1.00<2.4$ & Deficiente \\
\hline
\end{tabular}

Fuente: Salinas, et al. (2019)

Cabe destacar que, para determinar la relación entre las variables competencias docentes y rendimiento académico, se aplicó el coeficiente de correlación de Pearson, con el fin de alcanzar el objetivo general del estudio. Según Hernández et al. (2018), el coeficiente de Pearson es una prueba estadística que permite analizar la relación cuantitativa entre variables, medidas en un nivel por intervalo de razón. La fórmula utilizada para calcular el coeficiente de correlación de Pearson es la siguiente, considerando la posición de los autores ya referidos.

$$
\partial x y=\frac{\Sigma \mathrm{Dx} \cdot \mathrm{Dy}}{\sqrt{\Sigma D x^{2} \cdot D y^{2}}}
$$

Dónde:

$\partial x y=$ Coeficiente de correlación

$\sum$ Dx.Dy = Sumatoria del producto de Dx y Dy

$\sum D x^{2}=$ Sumatoria de $x^{2}$

$\sum D y^{2}=$ Sumatoria de $y^{2}$

De igual modo, para reflejar e interpretar el resultado derivado del cálculo del coeficiente de correlación, se muestra la tabla 3, donde se revelan el valor y la interpretación según los parámetros asumidos por Hernández et al. (2018); de este modo, el signo indica la dirección de la correlación (positiva o negativa), y el valor numérico la magnitud de la correlación. Del mismo modo, el programa computacional de análisis estadístico SPSS versión 19 expone de manera confiable los signos y valores correspondientes al cálculo, de acuerdo con lo manifestado por los autores.
Tabla 3. Baremo para la correlación

\begin{tabular}{cl}
\hline Valor & Interpretación \\
\hline$-0,90$ & Correlación negativa muy fuerte. \\
\hline$-0,75$ & Correlación negativa considerable. \\
\hline$-0,50$ & Correlación negativa mediana. \\
\hline$-0,25$ & Correlación negativa débil. \\
\hline$-0,10$ & Correlación negativa muy débil. \\
\hline$+0,00$ & No existe correlación alguna entre las variables. \\
\hline$+0,10$ & Correlación positiva muy débil. \\
\hline$+0,25$ & Correlación positiva débil. \\
\hline$+0,50$ & Correlación positiva mediana. \\
\hline$+0,75$ & Correlación positiva considerable. \\
\hline$+0,90$ & Correlación positiva muy fuerte. \\
\hline$+1,00$ & Correlación positiva perfecta. \\
\hline
\end{tabular}

Fuente: Hernández y Baptista (2016)

\section{RESULTADOS Y DISCUSIÓN}

Este proceso se hace en base en la presentación de los resultados estadísticos por cada una de las dimensiones e indicadores, respecto a la variable de la investigación. Los datos obtenidos se muestran en forma de frecuencias relativas o porcentajes, así como en medias aritméticas, en tablas elaboradas para tal fin. Es importante destacar que el análisis se efectuó agrupando los datos de acuerdo con las dimensiones que conforman dicha variable.

Este proceso se cumplió, trasladando los datos cualitativos, a través de datos cuantitativos expresados en las cuarenta y cinco (45) opiniones recopiladas según la respuesta de cada individuo, las cuales se reflejan en tablas de tabulación elaboradas para describir en ellas las frecuencias porcentuales.

a) Variable: Reflexión del Docente Universitario con Dimensión: Tipo de Conocimiento.

\section{Reflexion del docente Universitario}

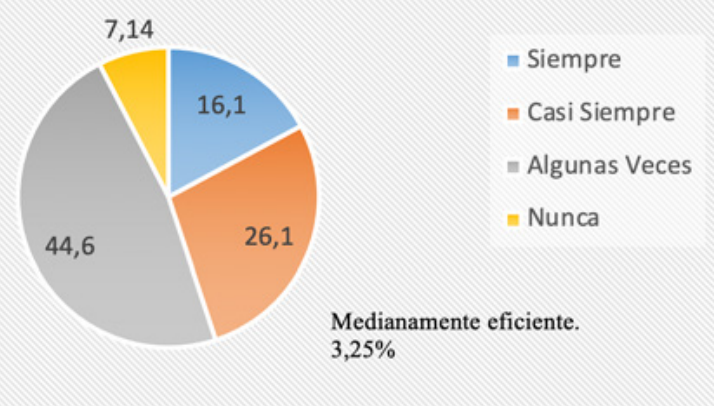

Figura 1. Ítems agrupados del 1 al 18 Fuente: Salinas et al. (2019) 
Tabla 4. Resultados generales de los ítems estudiados

\begin{tabular}{|c|c|c|c|c|c|c|c|c|c|c|c|c|}
\hline \multirow[b]{3}{*}{ ITEM } & \multicolumn{12}{|c|}{ ALTERNATIVAS } \\
\hline & \multicolumn{2}{|c|}{ Siempre } & \multicolumn{2}{|c|}{$\begin{array}{c}\text { Casi } \\
\text { Siempre }\end{array}$} & \multicolumn{2}{|c|}{$\begin{array}{c}\text { Algunas } \\
\text { Veces }\end{array}$} & \multicolumn{2}{|c|}{$\begin{array}{l}\text { Casi } \\
\text { Nunca }\end{array}$} & \multicolumn{2}{|c|}{ Nunca } & \multicolumn{2}{|c|}{ Total } \\
\hline & FA & FR & $\mathrm{FA}$ & FR & FA & FR & FA & FR & FA & FR & $\mathrm{FA}$ & FR \\
\hline $\begin{array}{l}\text { Escrito o } \\
\text { verbal }\end{array}$ & 13 & 23,20 & 9 & 50,00 & 6 & 26,80 & 7 & 0 & 10 & 0,00 & 45 & 3.26 \\
\hline Analítico & 5 & 0,00 & 8 & 19,60 & 11 & 37,50 & 16 & 42,90 & 5 & 0,00 & 45 & 3.20 \\
\hline Critico & 6 & 10,70 & 11 & 41,10 & 4 & 48,20 & 14 & 0 & 10 & 0 & 45 & 3.23 \\
\hline TOTAL & 35 & 26,00 & 25 & 43,00 & 21 & 32,00 & 29 & 0,00 & 25 & 0,00 & 135 & 3.25 \\
\hline
\end{tabular}

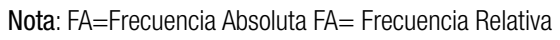
Fuente: Salinas, et al. (2019).

En estos resultados, se evidencia que las reflexiones del docente según la opinión de los estudiantes se sitúan en una categoría medianamente eficiente con el rango 3.25\%. Constatando los resultados con la teoría, se confirma lo expresado por autores como Bustamante, et al. (2018), para quienes los conocimientos se consolidan con la experiencia hasta llegar a la complejidad y se convierte en factor legitimador de ese aprendizaje. Por lo cual, estos conocimientos son mecanismo esencial dentro del accionar del profesor para la consolidación o fortalecimiento del pensamiento complejo.

b) Variable: Pensamiento Complejo y Dimensión: Tipos de Pensamientos.

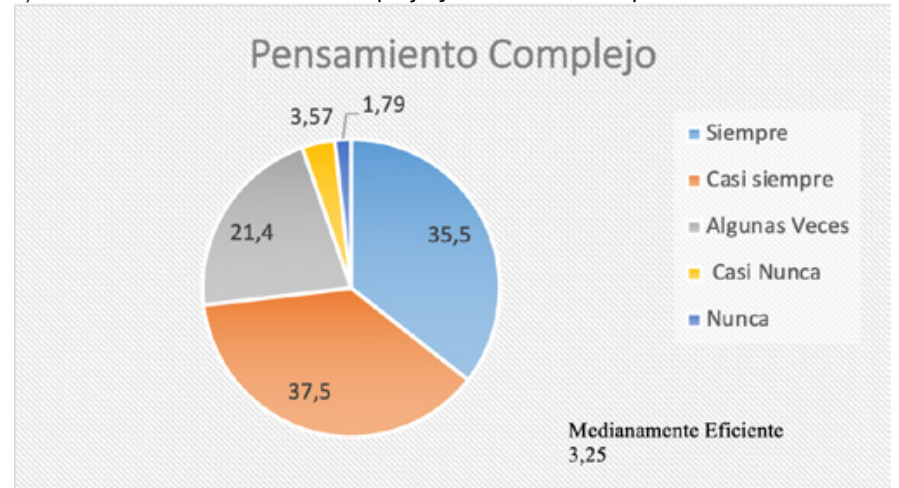

Figura 2. Ítems agrupados del 19 al 36 Fuente: Salinas, et al, (2019).

Tabla 5. Resultados generales de los ítems estudiados

\begin{tabular}{|c|c|c|c|c|c|c|c|c|c|c|c|c|}
\hline \multirow[b]{3}{*}{ ITEM } & \multicolumn{12}{|c|}{ ALTERNATIVAS } \\
\hline & \multicolumn{2}{|c|}{ Siempre } & \multicolumn{2}{|c|}{$\begin{array}{c}\text { Casi } \\
\text { Siempre }\end{array}$} & \multicolumn{2}{|c|}{$\begin{array}{c}\text { Algunas } \\
\text { Veces }\end{array}$} & \multicolumn{2}{|c|}{$\begin{array}{c}\text { Casi } \\
\text { Nunca }\end{array}$} & \multicolumn{2}{|c|}{ Nunca } & \multicolumn{2}{|c|}{ Total } \\
\hline & $\mathrm{FA}$ & $\mathrm{FR}$ & $\mathrm{FA}$ & FR & $\mathrm{FA}$ & FR & $\mathrm{FA}$ & $F R$ & $\mathrm{FA}$ & FR & FA & FR \\
\hline $\begin{array}{l}\text { Pensamiento } \\
\text { Metodológico }\end{array}$ & 9 & 16,10 & 6 & 26,80 & 13 & 44,60 & 8 & 7,14 & 9 & 5,36 & 45 & 3.23 \\
\hline $\begin{array}{l}\text { Pensamiento } \\
\text { Reflexivo }\end{array}$ & 12 & 35,70 & 7 & 37,50 & 10 & 21,40 & 3 & 3,57 & 13 & 1,79 & 45 & 3.25 \\
\hline $\begin{array}{l}\text { Pensamiento } \\
\text { Complejo }\end{array}$ & 16 & 16,10 & 8 & 8,93 & 4 & 25,00 & 10 & 46,40 & 15 & 3,57 & 45 & 3.25 \\
\hline TOTAL & 37 & 23,00 & 21 & 24,00 & 27 & 30,00 & 21 & 19,00 & 37 & 4,00 & 135 & 3.25 \\
\hline
\end{tabular}

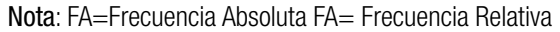

Fuente: Salinas, et al, (2019).

Según los resultados para la variable Fortalecimiento del Pensamiento Complejo del estudiante, los resultados obtenidos según los estudiantes encuestados se sitúan en medianamente eficiente (con el 3.25\%). Contrastándolo con Solana, (2005), citado en Núñez et al. (2017), "el pensamiento complejo deriva o emerge de los diversos tipos de pensamiento". De igual forma, se ve el mundo como un todo, es decir, este no se concibe de forma parcializada, así como tampoco el pensamiento complejo se visualiza en fragmentos. Por ello, queda evidenciado que la teoría difiere un poco con la realidad en el accionar teórico y práctico que manejan los estudiantes de la maestría de gerencia educativa.

\section{CONCLUSIONES}

Dando correspondencia a los indicadores establecidos dentro de la investigación, se concluye ciertos elementos que suscitan la pertinente reflexión:

Los docentes universitarios poseen y manejan conocimientos holísticos e integrales; sin embargo, se consideró pertinente que esto debe estar acompañado de actividades 0 acciones menos tradicionales, de modo que el saber llegue a los estudiantes. Del mismo modo, se concluyó que el conocimiento teórico academicista presenta divergencias entre lo que se proyecta dentro de las aulas de clase a nivel superior y con la realidad que se vive. Los estudiantes de la maestría en docencia superior requieren de la aplicación de estrategias innovadoras para desarrollar el pensamiento metodológico y reflexivo hasta llegar a la complejidad de los hechos.

En cuanto al dominio del pensamiento complejo, quedó evidenciado que los participantes de la maestría en docencia superior no han alcanzado la integralidad del pensamiento, dado que aún ven disgregado el conocimiento y les cuesta verlo como el todo de las partes.

Es necesario que el docente universitario, dentro de su accionar práctico, promueva los procesos cognitivos, pero de forma significativa, relevante e innovadora. De allí que la incorporación de nuevas estrategias sea tan necesaria para poder consolidar el proceso de análisis, síntesis, concreción del pensamiento y por ende la producción efectiva del aprendizaje.

Bajo el mismo espectro, es importante que el docente universitario establezca estrategias que conlleven a la resolución de conflictos dentro y fuera de los esquemas teóricos. Dichas estrategias contribuirían significativamente para formar estudiantes con competencias vinculantes a los contextos reales.

Se concluye, infiriendo que los docentes deben estar comprometidos a través de sus saberes teóricos-prácticos, de forma que puedan generar competencias analíticas, críticas, escriturales, mediante las que se pueda por ende llegar a pensamiento complejo en los participantes que cursan estudios de cuarto nivel.

En relación con el fortalecimiento del pensamiento, se recomienda una estructura cognitiva cada vez más equilibrada y compleja, ya que esta posee un cierto carácter universal. Puesto que esto permitirá reconocer la educación como un ente axiológico que contempla una serie de objetivos y contenidos propuestos a través del pensamiento cognitivo, analítico, crítico, hasta llegar a la complejidad y el holismo.

Se recomienda considerar la cotidianidad del quehacer del docente universitario, el cual en numerosas oportunidades no permite al participante reflexionar y accionar sobre su labor, sin hacer por tanto explícito su deber como investigador en formación.

De igual forma, se les exhorta a los docentes del nivel superior a abordar la temática sobre la reflexión de su accionar académico, con el fin único de que aborden estrategias innovadoras que les permita desarrollar en los maestrantes un pensamiento complejo e integrativo. 


\section{REFERENCIAS BIBLIOGRÁFICAS}

Bustamante, L. Ayllón, S \&, Escanés. G. (2018). Abordando la trayectoria universitaria desde el pensamiento complejo. Praxis Educativa (Arg), vol. 22, núm. 3, Universidad Nacional de La Pampa, Argentina.

Carlino, Paula (2002). Enseñar a escribir en la universidad: cómo lo hacen en Estados Unidos y por qué. Revista Iberoamericana De Educación, 12, 1-16.

Facione, Peter (1990). "Critical thinking: a statement of expert consensus for purposes of educational assessment and instruction", an American Philosophical Association, California, The California Academic Press, pp. 1-111.

Hernández, Sampieri, R. \& Mendoza, C (2018). Metodología de la investigación. Las rutas cuantitativa, cualitativa y mixta, Ciudad de México, México: Editorial Mc Graw Hill Educación, ISBN: 978-14562-6096-5, $714 p$

Hernández, R. \& Baptista, P. (2016). Metodología de la Investigación. (6 ed.) México: Mc Graw Hill.

Morin, E. (2010) Complejidad restringida, complejidad general. En: Revista estudios, VIII, (93): 81-135

Núñez, S, Ávila, E, \& Olivares, L. (2017). El desarrollo del pensamiento crítico en estudiantes universitarios por medio del Aprendizaje Basado en Problemas. Revista iberoamericana de educación superior, 8(23), 84-103.

Olivares, L, \& Heredia, Y. (2012). Desarrollo del pensamiento crítico en ambientes de aprendizaje basado en problemas en estudiantes de educación superior. Revista mexicana de investigación educativa, vol, núm. 17(54), 759-778.

PRELAC (2013), publicado por la Oficina Regional de Educación para América Latina y el Caribe (OREALC/UNESCO Santiago). Producción editorial: Ediciones del Imbunche.pp.4

Rivera, G, Espinosa M., \& Valdés D. (2017). La investigación científica en las universidades ecuatorianas: Prioridad del sistema educativo vigente. Revista Cubana de Educación Superior, 36(2), 113-125.

Salinas, R. Tesis Doctoral (2019), Saberes Pedagógicos como Elemento Potenciador en el Desarrollo de Pensamiento en el niño Innovador de Educación Inicial. Universidad Nacional Experimental Politécnica De La Fuerza Armada Nacional. pp.125 